\title{
ACCESIBILIDAD Y DOTACIÓN DE INFRAESTRUCTURA DE TRANSPORTE EN INMEDIACIONES DEL CAMPUS RESISTENCIA -UNNE-
}

\section{ACCESIBILITY AND ENDOWMENT OF INFRAESTRUCTURE OF TRANSPORT IN CONTIGUITY OF CAMPUS RESISTENCIA -UNNE-}

Prof. Alejandro Parras - Prof. Osvaldo Daniel Cardozo

Departamento de Geografía - Facultad de Humanidades - Universidad Nacional del Nordeste odcardozo@hum.unne.edu.ar

PALABRAS CLAVES: Universidad Nacional del Nordeste - Campus Resistencia - Accesibilidad Infraestructura de Transporte

KEY WORDS: Universidad Nacional del Nordeste (the University in the North-east of Argentina) Campus Resistencia - Accesibility - Infraestructure of Transport

\section{RESUMEN:}

La accesibilidad a los centros de actividad (oficinas públicas, fábricas, escuelas, centros de compras, etc) en el interior de una ciudad han despertado el interés de numerosos profesionales y estudiosos, dedicados a planificar la dotación y optimizar el uso de las infraestructuras -públicas y privadasrelacionadas con el transporte que canalizan la necesidad de desplazamiento de la población. Entendiendo la importancia conferida a esta cuestión y que es el camino más efectivo hacia la sustentabilidad de los recursos, la propuesta se centra en el análisis de la accesibilidad al Campus Resistencia dependiente de la Universidad Nacional del Nordeste -UNNE-, a partir de la descripción de las infraestructuras existentes en torno al mismo.

\section{ABSTRACT:}

The accessibility of the activity centres (public buildings, factories, schools, shopping areas, etc.) in the inner sections of a city has risen the interest of a number of professionals and scholars who ar dedicated to planning the endowment and optimization the usage of the infraestructure, either public or private, related to transport facilities that channel the travelling needs of the population. Given the importance of this particular issue and the most effective way towards the sustainability of the resources, the proposal is focused on the analysis of the accessibility to the Resistencia's Campus, dependent of the Universidad Nacional del Nordeste (the University in the North-east of Argentina), taking as a starting point a description of the infraestructure that already exist around it.

\section{SUMARIO:}

1. Introducción

2. Objetivo y Antecedentes

3. Técnicas y Método de Trabajo

4. Presentación de Resultados

5. Consideraciones Finales

6. Bibliografía

7. Notas 


\section{Introducción}

Los transportes se han convertido en la herramienta que permiten superar la fricción que suponen las distancias que separan a la población de los diversos centros de actividad, y es que no hay prácticamente ninguna actividad que sea posible sin la existencia del transporte (Barbero y Quinn, 1986: 127). Vinculada a ello, la dotación de infraestructuras y su distribución sobre el territorio determinan en gran medida la accesibilidad física a un lugar determinado. Por otra parte, la demanda y dotación de tales infraestructuras, están sujetas al uso del suelo que predomine en el lugar y a la atracción que ejerza el mismo sobre su entorno.

En los últimos años, la accesibilidad a los centros de estudio ha cobrado relevancia en el marco de la planificación urbana, siendo hoy considerada como un elemento de alta sensibilidad al momento de decidir sus emplazamientos. Esto se debe a las fuertes connotaciones que conlleva el factor localización -distancia, tiempo, dinero- para un sector de la población, como el de los estudiantes, quienes mayormente no trabajan o lo hace de manera informal.

Precisamente, esta situación se contrapone con la tendencia generalizada que aconseja relocalizar hacia la periferia a los edificios educativos ya existentes -o los nuevos por construir-, alejados del centro de las ciudades donde la competencia por las infraestructuras y los problemas de congestión del tránsito son mayores. El emplazamiento de un centro de educación en cualquiera de sus niveles debido a la fuerte atracción diaria de población que genera, no haría más que potenciar dichos problemas en el casco céntrico de una ciudad, aunque como se afirmó antes, esto conlleva un aumento en los presupuestos de tiempo y dinero para desplazarse.

Este es el caso de los campus universitarios, quienes debido a las variadas ofertas académicas que reúnen se convierten en verdaderos complejos edilicios integrados por un conjunto de facultades e institutos. Su actividad atrae diariamente a una población de varios miles de personas entre alumnos, profesores y personal no docente; atender satisfactoriamente las necesidades de movilidad y accesibilidad de esta población, hace necesario la adopción de criterios de planificación en la dotación de las infraestructuras de transporte con el fin de racionalizar y optimizar en el uso de los mismos.

La estrecha relación existente entre la accesibilidad física a un lugar y las infraestructuras relacionadas con el transporte y la movilidad -calles, avenidas, paradas de transporte, etc.-, pone en evidencia la importancia conferida al conocimiento y la disponibilidad de esta información para la planificación y toma de decisiones, sobre todo si consideramos que una accesibilidad plena es decisiva para mejorar la calidad de vida de las personas.

\section{Objetivo y Antecedentes:}

Considerando que la función educativa de la Universidad Nacional del Nordeste -UNNE- ejerce una importante atracción sobre las provincias del Chaco, Corrientes, Formosa, norte de Santa Fe y Misiones, como así también, sobre buena parte del territorio nacional ${ }^{(1)}$, y debido a la particular influencia que posee sobre las ciudades de Resistencia y Corrientes -las dos sedes principales-, se impone la necesidad de contar con información cartográfica disponible en internet referida a la accesibilidad a los campus de la Universidad en dichas ciudades.

En este sentido y teniendo en cuenta que la Universidad reparte sus funciones académicas en tres ciudades (Corrientes, Resistencia, y Saénz Peña), en una primera instancia se toma como referencia espacial al Campus Resistencia, para realizar un análisis descriptivo de su accesibilidad a partir del conjunto de infraestructuras vinculadas al servicio de transporte existentes en torno al mismo, con el fin subsiguiente de elaborar cartografía analítica y de síntesis destinada a su incorporación en la página web de la Facultad Humanidades y la Universidad.

Como referencias de emprendimientos similares pueden mencionarse varios trabajos. Entre ellos Bullock-Dickens-Steadman (1975) en su obra sobre la estructura de los espacios urbanos, presentan desde una óptica netamente teorética, un capítulo referido al análisis y planeamiento de las actividades diarias en una universidad estadounidense. 
Por otra parte, el aporte español es el más numeroso y de ellos, la obra de Gómez Mendoza et al (1986) circunscripta a la Universidad Autónoma de Madrid, dedica un capítulo a los transportes que sirven al Campus, y donde se exponen algunos datos de interés respecto a la movilidad del estudiantado, además de la oferta de infraestructura de transporte. También Brunet Estarelles y Gálvez Capellá (1995) se ocupan de estudiar la movilidad en el Campus de la Universitat de les Illes Balears, generado a partir de un censo de ingreso/egreso vehicular.

Más recientemente, Miralles Guasch et al (2002), emprenden un trabajo referido específicamente al desplazamiento cotidiano de los alumnos que concurren al Campus Bellaterra de la Universidad Autónoma de Barcelona. Por su parte, investigadores del Instituto Universitario de Geografía de la Universidad de Alicante, han generado un SIG (Sistema de Información Geográfica) destinado -entre otros diversos usos- a gestionar la accesibilidad a su campus universitario.

En el ámbito local se reconocen varios documentos (Cardozo-Rey-Foschiatti, 2001/2002 y Cardozo, 2002) relacionados al análisis de, la incidencia del uso de los transportes en la calidad de vida de los estudiantes que concurren al Campus Resistencia de la Universidad Nacional del Nordeste -UNNE-.

\section{Técnicas y Método de Trabajo:}

Dentro del conjunto de tareas desarrolladas es posible identificar dos grandes conjuntos, las relacionadas con el trabajo de gabinete -en el Departamento de Geografía- y las realizadas como parte del trabajo de campo o salida al terreno ${ }^{(2)}$. De entre las actividades más relevantes caben mencionar las siguientes:

- Consulta de bibliografía específica referida a temas como: servicios y territorio, dotación de servicios urbanos, servicios e infraestructuras relacionados al transporte, cartografía de accesibilidad.

- Búsqueda en internet de ejemplos cartográficos referidos a la accesibilidad en centros de estudio o campus universitarios.

- Determinación de los límites del área a estudiar, y definición de la profundidad con que se aborda el tema (descriptivo).

- Identificación, selección y jerarquización de los elementos del terreno a incluir en el relevamiento.

- Una vez identificados y seleccionados los elementos a relevar se confeccionó una "planilla de registro" codificada en forma alfa-numérica, con el objeto de organizar la recolección de la información y facilitar su posterior ingreso a la base de datos.

- Obtención y preparación de la cartografía base: se obtuvo un plano de la ciudad en formato DWG perteneciente a la Dirección de Catastro de la Municipalidad de Resistencia, lo que hizo necesario su posterior transformación a un formato de tipo SHP.

- En el proceso de transformación de formatos (DWG a SHP) se produjo la pérdida de información, referida a ciertas manzanas en torno al Campus y de otras entidades mayores de tipo parcelarias, por lo que debió completarse la base cartográfica a través de la digitalización de nuevos polígonos.

- Definición de los parámetros de la presentación cartográfica final del área en estudio. Se decidió que la de tipo temática estará referida específicamente a cada una de las variables consideradas (área de estudio, red vial, paradas de transporte, señalización, etc.), en tanto que la de síntesis contendría solo la información mínima indispensable -principal y necesaria- con el propósito de no sobrecargar la representación, es decir:

- Las paradas de transporte, discriminadas según modo de transporte

- Las avenidas que la circundan y sus respectivos nombres, número postal e indicaciones del destino más próximo o importante

- Los puntos o sitios de acceso al Campus Resistencia

- Sumado a todos los elementos propios de una representación cartográfica (título, norte geográfico, escala, referencias) 
- Las tareas de campo propiamente dichas incluyen una serie de actividades cumplidas recorriendo el área de estudio: el mismo se realizó en dos etapas principales y otras complementarias de menor envergadura, abarcándose en total un espacio de aproximadamente 40 manzanas (Ver Fig. 1).

- Concluida la etapa de trabajo sobre el terreno, se planificó la simbolización -colores, tamaños y formas- para representar los elementos del terreno.

- Luego, como parte de una de las principales labores de gabinete, se procedió a la digitalización de los elementos del terreno (puntos, líneas y polígonos), y la carga de sus respectivos atributos.

- Concluida la fase de digitalización, se procedió a la elaboración de cartografía analítica y de síntesis. Para ello se emplearon distintos software específicos (Arc View 3.1, Paint, Corel Draw 8 y Corel Photo Pain 8).

- Finalmente se efectuaron pruebas de exportación (en distintos formatos: CDR, BMP, JPG, GIF), la edición de las representaciones(salida gráfica), y varios test de control.

\section{Presentación de Resultados:}

Se trata de un Campus universitario localizado en el sector sudoeste de la ciudad de Resistencia (Ver Fig. 2), capital de la Provincia del Chaco, concretamente en intersección de las avds. Las Heras y Juan José Castelli. Este complejo de educación superior reúne en su interior -unas 8 has. de superficie en total- a diversas facultades (Arquitectura y Urbanismo, Ciencias Económicas, Humanidades, Ingeniería) e institutos (Medicina Regional, Biblioteca Central, Farmacia, Obra Social), lo cual supone una población flotante diaria estimada en unas 18.000 personas aproximadamente (Cardozo, 2002: 7).

\section{Fig. 1 Área Metropolitana del Gran Resistencia Localización del Campus Resistencia -UNNE-}

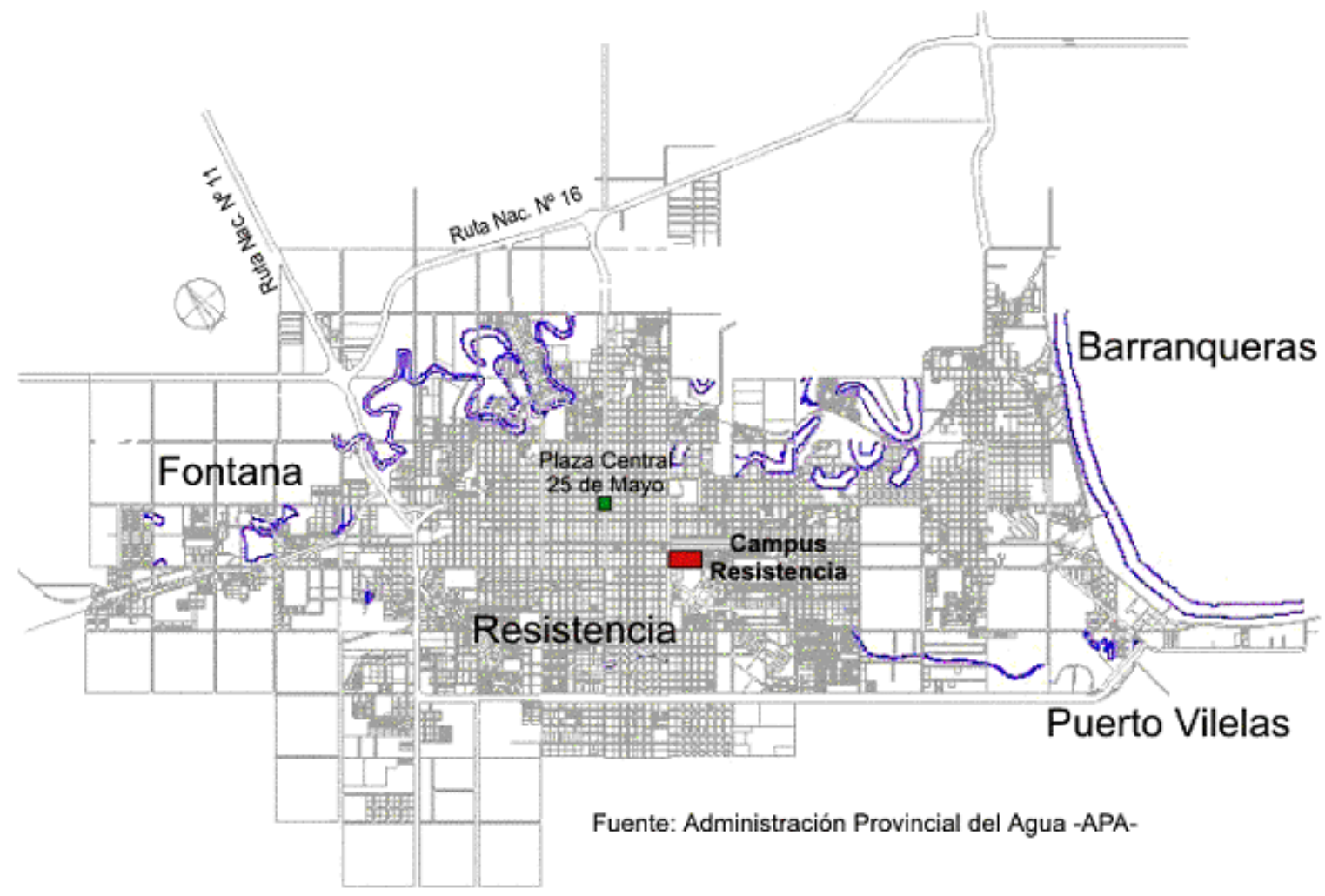




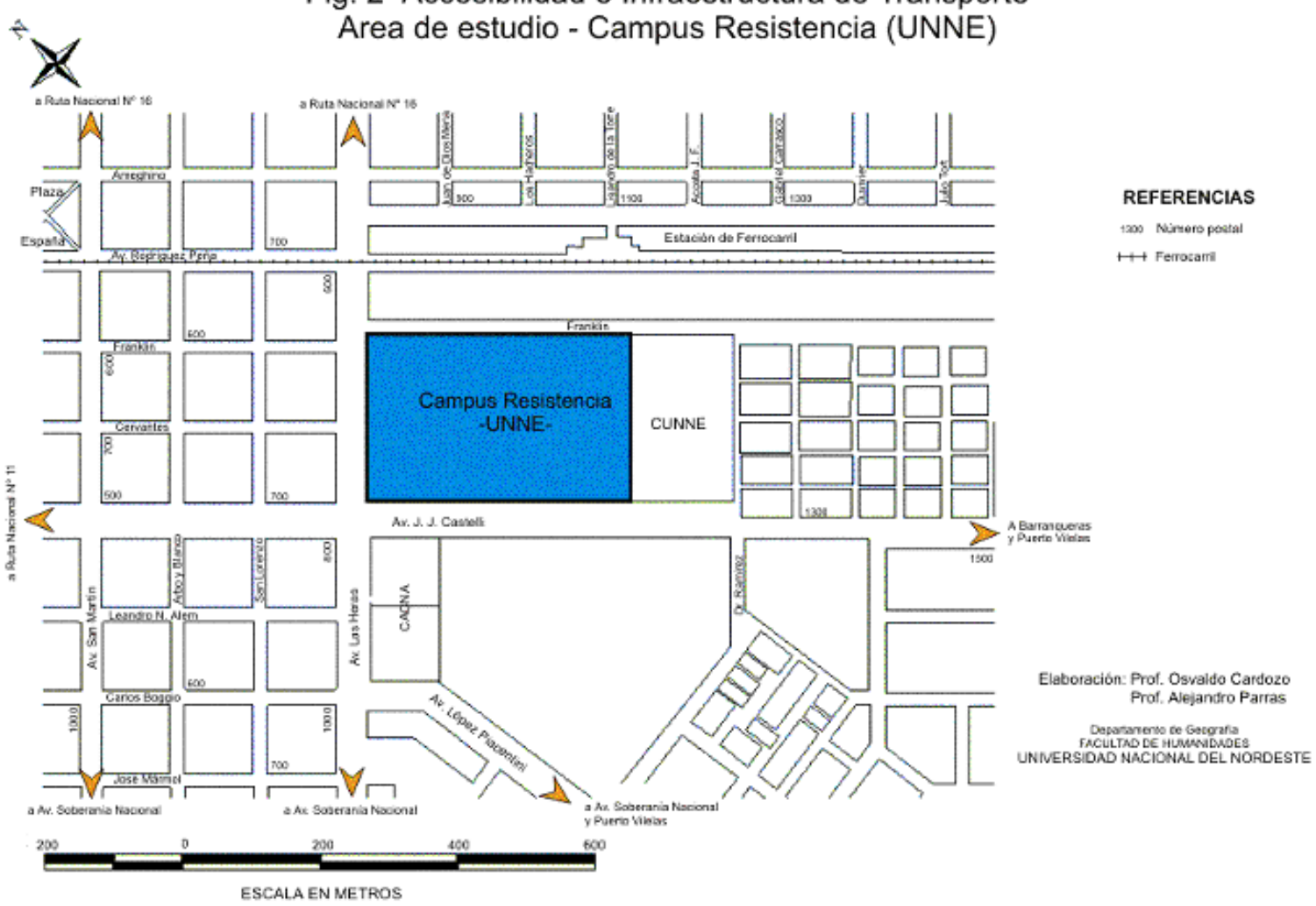

A continuación se presenta el análisis de la cartografía temática referida a cada una de las variables consideradas en el estudio de la accesibilidad al Campus Resistencia.

Red Vial: su estudio se realizó en base a dos características consideradas relevantes para análisis de la accesibilidad: la jerarquía (avenida, calle, pasaje), y el tipo de calzada que presenta (asfalto, ripio, tierra), representadas en las Fig. 3 y 4. Con respecto a la primera, el acceso principal al Campus está servido por una avenida de tres carriles (Las Heras), en tanto que los accesos secundarios se ubican sobre una avenida de dos carriles (Juan José Castelli) y una calle (Franklin); tomando en consideración su punto de acceso principal sobre la Av. Las Heras, a 300 metros es posible acceder a otras dos avenidas (San Martín y López Piacentini) de dos carriles.

Fig. 3 Accesibilidad e Infraestructura de Transporte

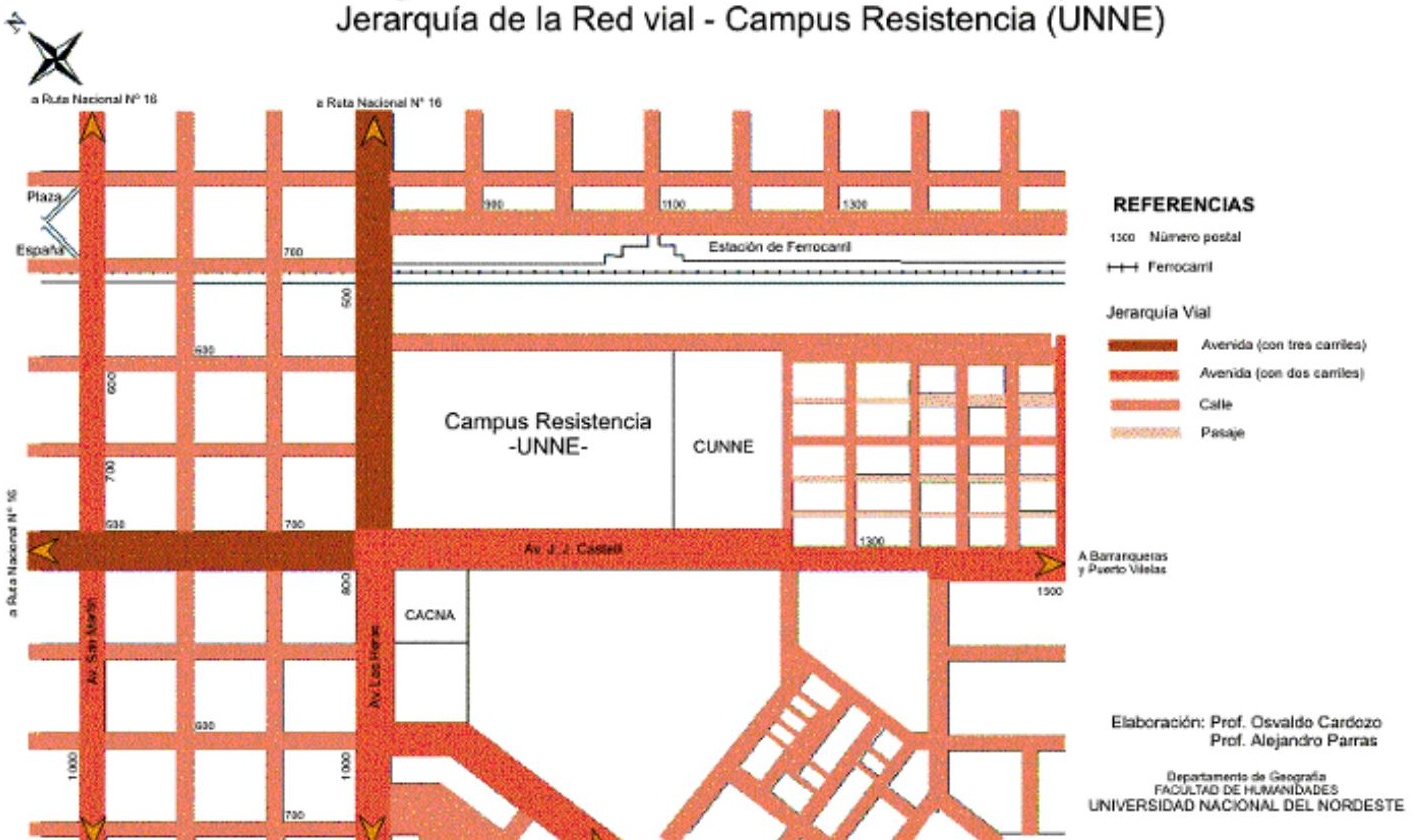


En relación al tipo de calzada que presenta la red vial, se advierte un predominio de calzadas con cobertura asfáltica por sobre las otras. La cobertura con ripio es la menos numerosa y solo se localizan en el sector Sudoeste, sobre la Av. López Piacentini, un tramo de la Av. Las Heras, y la calle Dr. Ramírez. Las calles que permanecen sin ninguna cobertura (de tierra), aunque más numerosas que las de ripio, solo se restringen al sector sudeste.

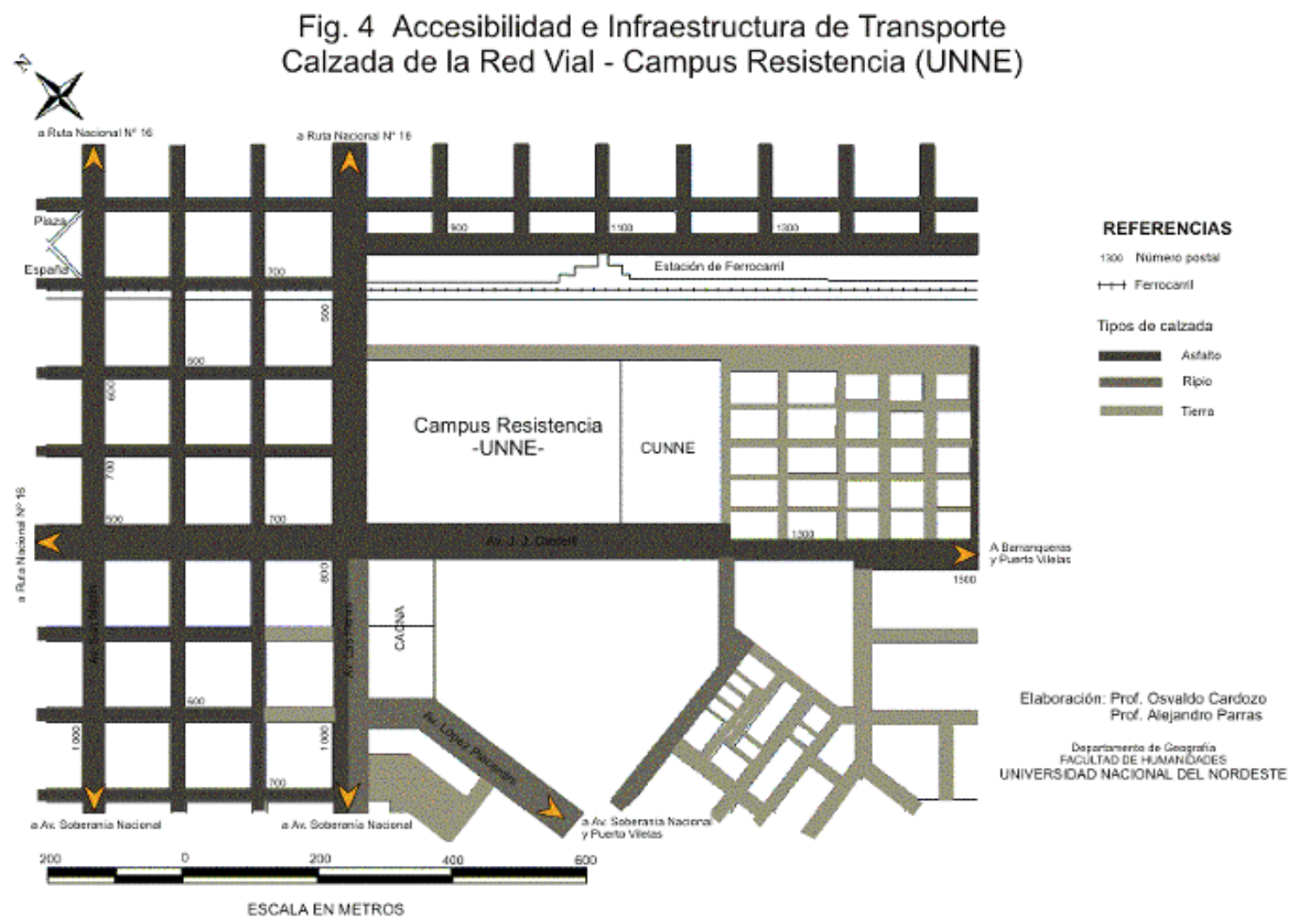

Accesos: el Campus Resistencia presenta varios sitios de acceso (Ver Fig. 5). El mayor número (4) se ubican sobre la Av. Las Heras, en tanto que sobre la Av. Juan José Castelli y calle Franklin se emplazan dos sobre cada una. Los ubicados sobre la Av. Juan José Castelli al igual que los emplazados sobre la calle Franklin, en la actualidad permanecen cerrados al tránsito, por lo tanto, el principal punto de ingreso y egreso al Campus Resistencia se halla sobre la Av. Las Heras: dos son de tipo vehicular (uno para el ingreso y otro para el egreso) y dos de tipo peatonal.

Cabe señalar que este último sitio, constituye un punto de continuas fricciones entre los usuarios de la vía publica, tanto en calidad de conductores como de peatones. Observaciones efectuadas en el lugar permitieron detectar numerosas situaciones ambientales (ubicación incorrecto del sitio de egreso) y culturales (caso omiso al semáforo, senda peatonal, cruce por las esquinas, etc) que dificultan la movilidad de las personas y los convierten en sujetos vulnerables a sufrir accidentes de tránsito.

Paradas de Transporte: la localización de las paradas de transporte muestra un claro desequilibrio en su distribución (Ver Fig. 6). La mayor parte de ellas se ubican hacia los sectores Norte y Oeste del Campus, fundamentalmente sobre las avenidas Las Heras y San Martín, en tanto que hacia el Sur y Este su número disminuye notablemente.

Como la "lógica espacial" lo indica, el mayor número y variedad de ofertas de transporte se centraliza en torno al acceso principal del Campus, con diversas paradas de remis, colectivo (urbano e interurbano) y combi, en un radio de 100 metros. Mientras que la oferta de transporte en ferrocarril (Sefecha) se localiza sobre la Av. Rodríguez Peña (paralela al tendido de las vías férreas) a 400 metros del acceso al Campus, la del transporte en colectivos lo hace sobre las avenidas Las Heras, San Martín y la calle Ameghino 


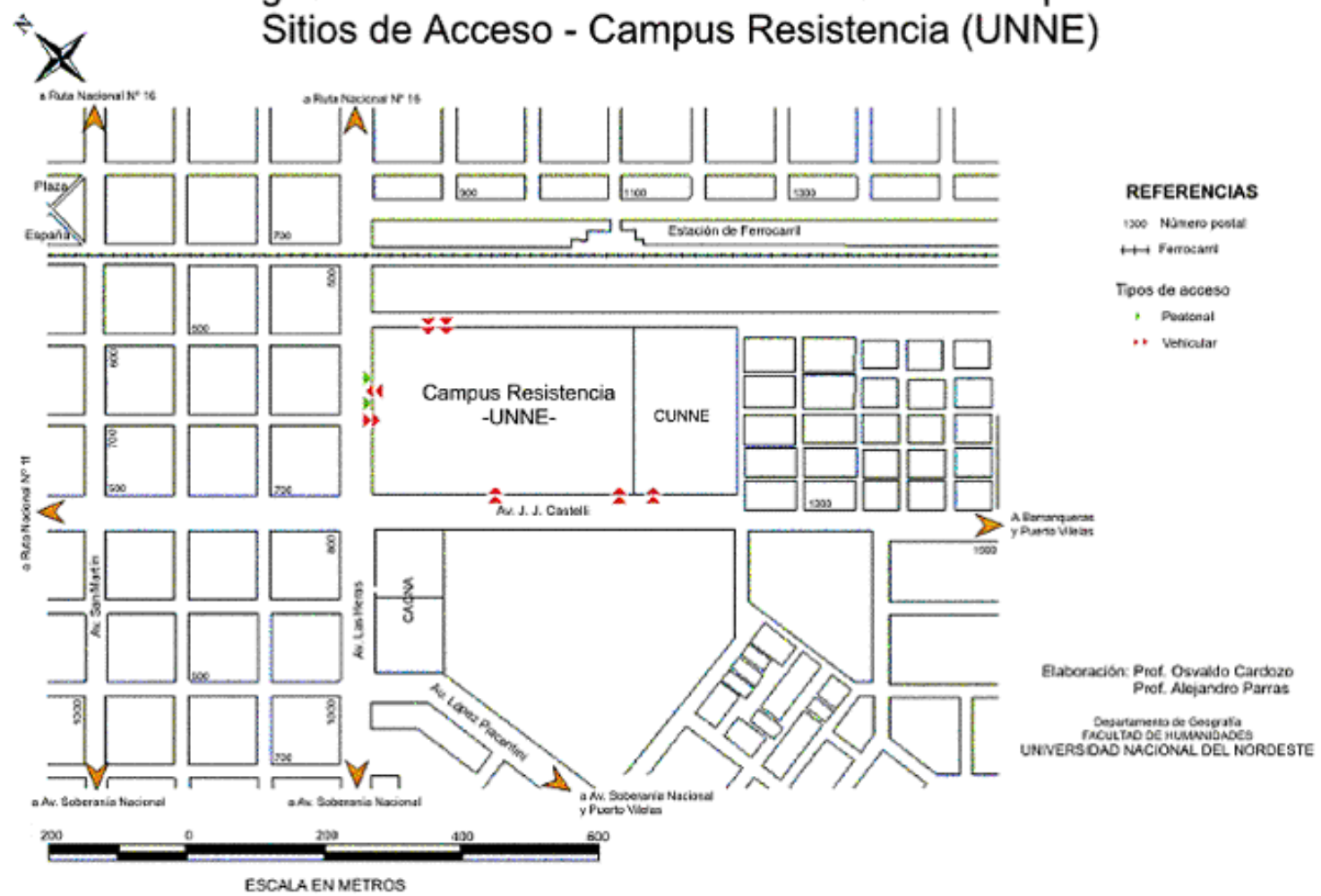

Fig. 6 Accesibilidad e Infraestructura de Transporte

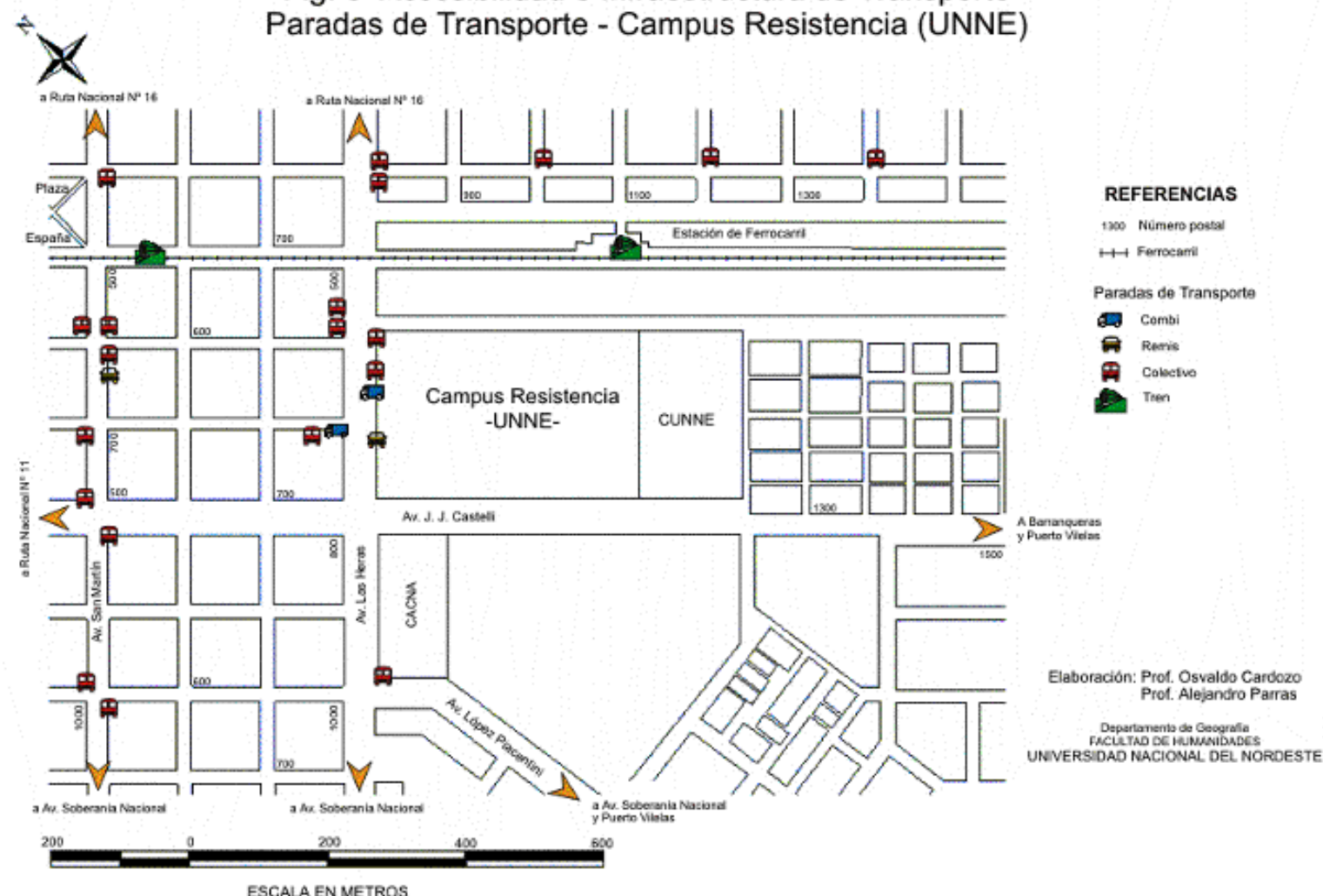

Señalización: la distribución espacial de las señales viales y de tránsito presenta una marcada concentración en el sector Norte de la representación (Ver Fig. 7), concretamente en torno a los accesos al Campus y las vías del Ferrocarril. La señalización de tipo horizontal (sendas peatonales) se agrupa mayormente en el frente del Campus -sobre la Av. Las Heras-, en tanto que la señalización de tipo 
vertical (carteles) se concentra en torno al tramo de las vías de ferrocarril comprendido entre las avenidas Las Heras y San Martín.

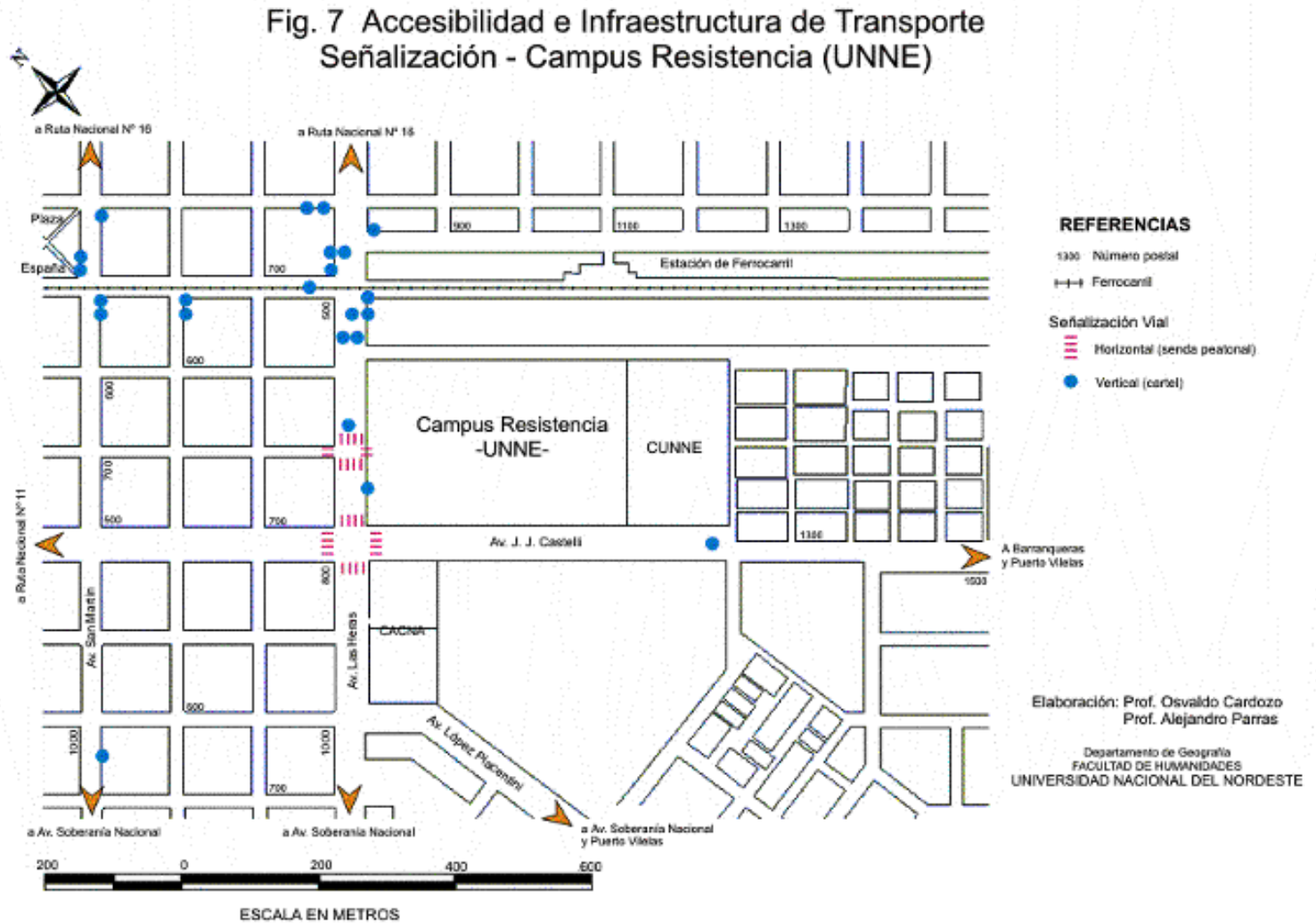

Semáforos: la distribución de los semáforos presenta una fuerte concentración espacial en determinados sitios del área en estudio (Ver Fig. 8): en el acceso (ingreso-egreso) principal al Campus, y en las intersecciones de Av. Juan José Castelli con la Av. San Martín y con la calle Dr. Ramírez; la presencia de semáforos en esta esquina, se debe a que constituye una de las principales vías de acceso al Barrio Llaponagat.

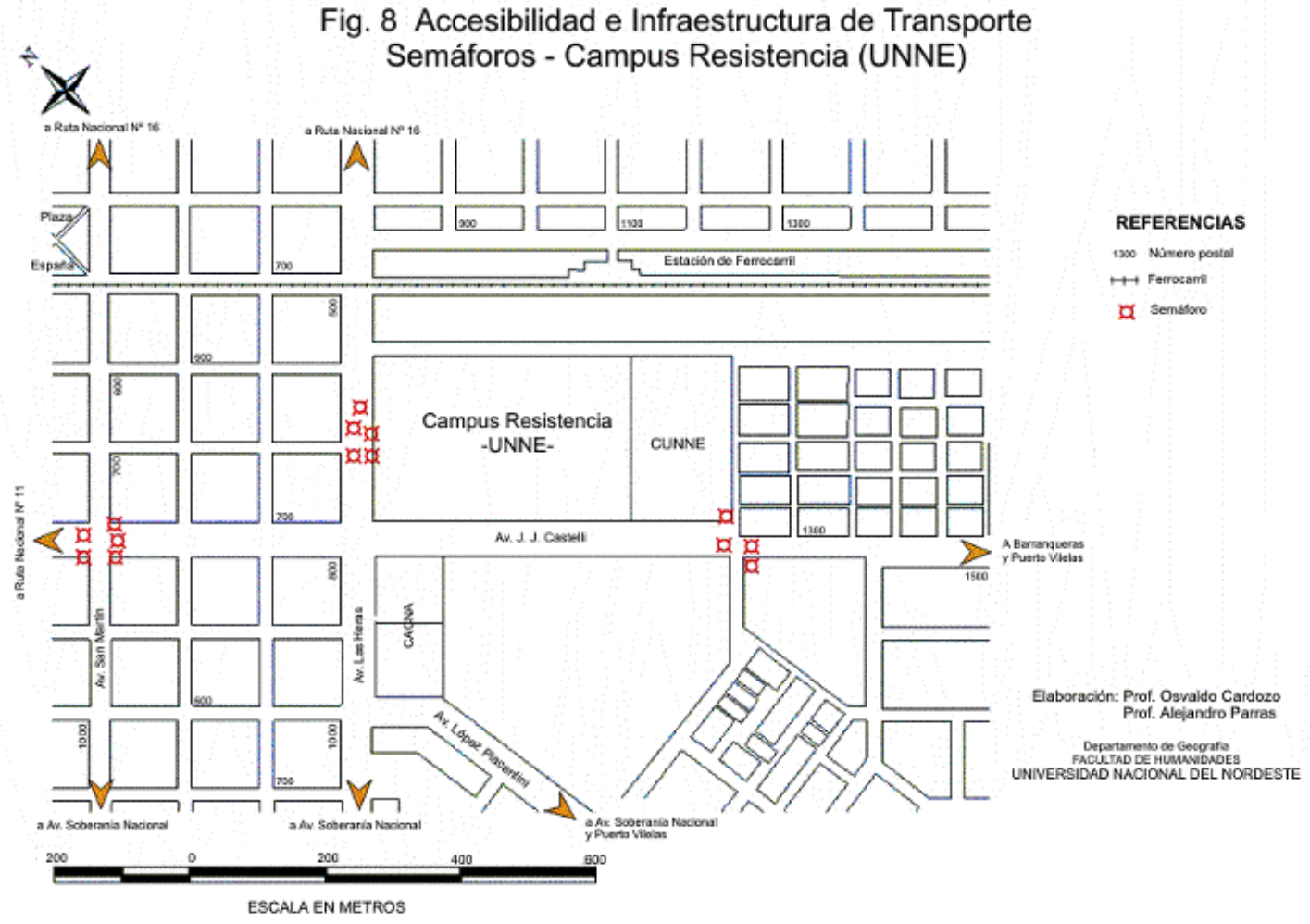




\section{Consideraciones Finales:}

El conocimiento a fondo del territorio -su limitaciones y potencialidades-, permite optimizar el uso de los recursos disponibles. En este plano de análisis, resulta evidente que la localización de los usos de suelo y su dotación de infraestructuras -sobre todo las de transporte- determinan la accesibilidad física a un lugar, independientemente de las condiciones de movilidad de la población. Sujeto a este marco empírico, se presentan las consideraciones preliminares de las principales variables empleadas en la caracterización de la accesibilidad al Campus Resistencia -UNNE-.

La red vial que circunda al Campus está compuesta por avenidas -de 2 y 3 carriles-, calles y pasajes. Las avenidas más importantes son Las Heras y Juan José Castelli, en cuya intersección se emplaza el Campus, en tanto que las avds San Martín y López Piacentini se encuentran a una distancia de 300 metros aproximadamente de su acceso principal; todas ellas poseen cobertura asfáltica, excepto un tramo de la Av. Las Heras y la Av. López Piacentini. La red de calles, mucho más numerosa cubre gran parte del espacio en estudio y solo dos cuadras de las calles Leandro N. Alem, Carlos Boggio y las del Barrio Llaponagat, no poseen asfalto. Con respecto a los pasajes solo se disponen al sudeste del Campus y son de tierra.

Los accesos más importantes y de uso cotidiano, se emplazan sobre la Av. Las Heras, en tanto que los ubicados sobre la Av. Juan José Castelli y la calle Franklin permanecen cerrados al tránsito. Por su parte, las paradas de transporte se reparten de una manera más homogénea, aunque denota una mayor concentración de variedad y cantidad sobre la Av. Las Heras frente al acceso principal del Campus; otros puntos sobresalientes se reconocen sobre la Av. San Martín y la calle Ameghino.

La distribución de la señalización muestra una marcada concentración en torno al acceso del Campus, y en la intersección de Av. Las Heras y las vías del ferrocarril. Con respecto a los semáforos, muestran una asignación espacial caracterizada por una fuerte concentración en la zona de acceso al Campus, y otros puntos menores sobre la Av. Juan José Castelli.

En general, la distribución de las infraestructura vinculadas al transporte y la accesibilidad al Campus, muestra espacios con marcadas concentraciones -en algunos casos ociosas-, y en otras con preocupantes vacíos. De todas maneras, la concentración sobre la Av. Las Heras, se justifica por la presencia de los accesos al Campus.

\section{Bibliografía:}

- Brunet Estarelles, Pere J., y Gálvez Capellá, Francisco, J. (1995). Estructura urbana y movilidad: análisis del tráfico inducido por el Campus de la Universitat de les Illes Balears. En: $\underline{\text { XIV }}$ Congreso Nacional de Geografía. Salamanca. Pp: 322-325.

- Bullock, Nicholas; Dickens, Peter; y Steadman, Philip (1975). La elaboración de modelos de actividades diarias. En: La Estructura del Espacio Urbano. Colecc. Ciencia Urbanística. Edit. GUSTAVO GIL, Barcelona. Pp: 187-224.

- Cardozo, Osvaldo D. (2002). El viaje hacia la Universidad de los estudiantes del Campus Resistencia -UNNE-. XXII ${ }^{\circ}$ Encuentro de Geohistoria Regional. Instituto de Investigaciones Geohistóricas - Conicet. Resistencia. 
IG Revista Geográfica Digital. IGUNNE. Facultad de Humanidades. UNNE. Año 2- No 3 Enero - Junio 2005. ISSN 1668-5180. Resistencia, Chaco.

- Cardozo, Osvaldo D.; Rey Celmira E.; Foschiatti, Ana M. (2001). Condiciones de transporte y calidad de vida: desplazamientos cotidianos de los estudiantes al Campus Resistencia UNNE-. Secretaría General de Ciencia y Técnica de la Universidad Nacional del Nordeste. Corrientes.

- Cardozo, Osvaldo D.; Rey Celmira E.; Foschiatti, Ana M. (2002). La movilidad por motivos de estudio: desplazamientos cotidianos hacia el Campus Resistencia -UNNE-. Secretaría General de Ciencia y Técnica de la Universidad Nacional del Nordeste. Resistencia.

- Gómez Mendoza, Josefina et al. (1986). Los transportes a la Universidad Autónoma de Madrid. (Cap. Quinto). En: Ghettos Universitarios. El Campus de la Universidad Autónoma de Madrid. Colecc. de Bolsillo. № 7. Departamento de Geografía. Ediciones de la Universidad Autónoma de Madrid -UAM-, Madrid. Pp: 147-173.

- Miralles Guasch, Carme et al. (2002). Las condiciones de la movilidad en un nodo de la ciudad metropolitana: el caso de la Universitat Autònoma de Barcelona. En: www.unileon.es/dp/dge/res-mir.html

- UNIVERSITAT D'AliCANTE. (1998). SIG del Campus de la Universitat d'Alicante. En: http://www.ua.es/secretaria.gral/es/memoria/1998 99/iv5.htm

- UNIVERSITAT D'ALICANTE. (2002). La Universidad de Alicante ha ultimado el Plan de Accesibilidad al Campus. En: http://www.ua.es/es/servicios/comunicacion/notas/03112002.html

\footnotetext{
Notas:

(1) Esto fue posible advertir en una plancha cartográfica inédita referida a la distribución de la matrícula de la Universidad Nacional del Nordeste sobre todo el territorio nacional. La misma fue elaborada con datos correspondientes a la matrícula del año 2001 , en el marco del ATLAS GEOGRÁFICOA DE LA PROVINCIA DE CORRIENTES, proyecto del Instituto de Geografía de la Facultad de Humanidades -UNNE-.

(2) Cave señalar que la mayor parte de la información necesaria para el cumplimiento de los objetivos propuestos no se encontró disponible, por lo que fue necesario generarla "in situ" a partir de varias salidas de campo.
} 